\title{
Reduction of Beta-Lactam Antimicrobial Activity in Staphylococcus aureus Abscesses by Neutrophil Alteration of Penicillin-Binding Protein 2
}

\author{
David M. Bamberger, Matthew Goers, Tim Quinn, Betty Herndon* \\ Departments of Medicine and Basic Medical Science, School of Medicine, University of Missouri, Kansas City, USA. \\ Email: "herndonb@umkc.edu
}

Received April $9^{\text {th }}, 2012$; revised May $8^{\text {th }}, 2012$; accepted Jun $7^{\text {th }}, 2012$

\begin{abstract}
We previously demonstrated that brief nonkilling neutrophil exposure diminishes the binding affinity of $S$. aureus penicillin-binding protein (PBP) 2. We sought to investigate further the role of the neutrophil in the alteration of antimicrobial activity and its interaction with PBP-2 by studying the activity of cefotaxime, which highly binds to PBP 2, and cephalexin, which minimally binds to PBP 2. Using S. aureus, cultured in vitro in sterile-filtered normal and neutrophil depleted abscess fluid, we sought to demonstrate an in vivo significance of the neutrophil effect upon the activity of antimicrobials that target PBP-2 by studying the same antimicrobials in an experimental S. aureus abscess. Rats were implanted with perforated tissue cages and infected with $S$. aureus; some rats were neutrophil depleted by mechlorethamine. Abscess fluids from normal and neutropenic abscesses were harvested, pooled, sterile-filtered and stored for the time-kill studies. Treatment studies were performed by administering either $300 \mu \mathrm{g} / \mathrm{kg} / \mathrm{d}$ cefotaxime or cephalexin for 7 days in other rats with 24 hour-old tissue-cage $S$. aureus abscesses. In time-kill studies, cefotaxime was highly active against stationary phase $S$. aureus in MHB and in neutropenic abscess fluid, but less active in the non-neutropenic ab- scess fluid ( $\mathrm{p}<0.05$ compared to neutropenic abscess fluid). Cephalexin was equally active in neutropenic and non- noneutropenic abscess fluids, and more active than cefotaxime in the abscess model after 7 days of therapy $\left(2.1 \pm 1.7 \log _{10}\right.$ kill, $\mathrm{p}=0.029$ vs. $\left.0.81 \pm 2.5, \mathrm{p}=\mathrm{NS}\right)$. These data suggest that neutrophil exposure, which diminishes $S$. aureus PBP-2 binding affinity [or total quantity], also adversely affects the antimicrobial activity of cefotaxime, which binds to PBP-2, as compared to cephalexin. Altered PBP targets from neutrophil exposure may be a mechanism of antimicrobial resistance within abscesses.
\end{abstract}

Keywords: Neutrophils; Penicillin-Binding Proteins; S. aureus; Abscesses

\section{Introduction}

The treatment of abscesses caused by Staphylococcus aureus usually necessitates drainage and antimicrobials in the setting of an abscess usually only have limited activity. However some abscesses respond to antimicrobial therapy without drainage, and the mechanisms by which antimicrobial activity is diminished in a suppurative environment are not fully understood, but are not likely related to inadequate antimicrobial concentrations [1]. We have found that the activities of antimicrobials within polymorphonuclear leukocytes (PMNs) correlated with in vivo efficacy in the treatment of experimental $S$. aureus abscesses [2]. The PMN may act a sanctuary since we have previously found that killing by PMNs, but not phagocytosis of staphylococci is inhibited systemically

\footnotetext{
"Corresponding author.
}

by the presence of an abscess, and may be further inhibited in the abscess milieu [3]. We have further shown that cefazolin is more effective in abscesses in which PMN influx was inhibited [4].

A significant virulence mechanism of $S$. aureus is its ability to survive within the PMN [5]. During the time of intracellular survival, global changes in $S$. aureus gene expression occur, including changes in the expression of penicillin-binding proteins (PBPs) genes [6]. PBPs are the targets of beta-lactam activity which inhibits transpeptidase activity of PBPs by forming a covalent penicilloylenzyme complex that blocks the normal transpeptidation reaction and results in bacterial death [7]. We have demonstrated that beta-lactam binding affinity to $S$. aureus PBP-2 is significantly decreased by brief non-killing PMN exposure, and postulated that this may be associated with diminished antimicrobial activity within an 
abscess milieu [4]. The diminished binding affinity observed may be secondary to decreased PBP-2 production or alteration of the target for beta-lactam binding $[7,8]$.

Cefotaxime a third generation cephalosporin and cephalexin a first generation cephalosporin, both have similar in vitro activity against $S$. aureus and similar pharmacokinetics, including serum half-life and protein binding. However, the PBP targets of the two agents differ. Cefotaxime binds selectively to PBP 1 and 2, and cephalexin binds to PBP 1 and 3, each at its respective minimum inhibitory concentration $[9,10]$. We sought to demonstrate that the detrimental role of the neutrophil in the alteration of antimicrobial activity is due to its effects on PBP-2 by studying the activity of cefotaxime, which highly binds to PBP 2, and cephalexin, which only minimally binds to PBP 2, against $S$. aureus in vitro in Mueller-Hinton broth (MHB), sterile filtered abscess fluid, and sterile filtered neutrophil depleted abscess fluid. We further sought to demonstrate an in vivo significance of the effect on neutrophils upon the activity of antimicrobials that target PBP-2 by studying the same antimicrobials in an experimental $S$. aureus abscess. We postulated that if the diminished beta-lactam binding affinity of $S$. aureus PBP 2 by PMNs has the potential for clinical significance, that cefotaxime, which binds PBP 2, would have diminished activity compared to cephalexin in vitro in sterile filtered abscess fluid and in the in vivo experimental abscess model, but not in vitro in MHB or in sterile filtered PMN depleted abscess fluid.

\section{Materials and Methods}

\subsection{Ex-Vivo Studies, Neutropenic and Normal Abscess Fluid Preparation}

Utilizing sterile techniques, ten rats were implanted with perforated tissue cages (gas sterilized table tennis balls with $3001.5 \mathrm{~mm}$ diameter holes, 1 per rat) and allowed to encapsulate intra-abdominally for 6 weeks [2]. One day before infection, six rats were administered $0.5 \mathrm{ml}$ mechlorethamine $\mathrm{HCl}$ [Sigma-Aldrich, St. Louis, MO] 1 $\mathrm{mg} / \mathrm{ml}$ in saline i.v. to deplete PMN and precursor cells. This agent - acts against bone marrow precursors of PMN giving a more complete and longer term depletion than anti-rat PMN antibody which we have used in the past. 24 $\mathrm{hr}$ following PMN depletion in the six rats, all ten capsules were inoculated with $1 \times 10^{7} \mathrm{cfu}$ of a Staphylococcus aureus isolated initially isolated from a bacteremic patient, and used in previous studies [2-4]. Three $\mathrm{mL}$ abscess fluid was removed by syringe aspiration from each infected capsule daily until at day 7 when as much fluid as possible was harvested at necropsy. The abscess fluids were sonicated at 90 watts for 15 seconds, sterile-filtered $\left(0.2 \mu \mathrm{M}\right.$ syringe filters) and stored at $-70^{\circ} \mathrm{C}$ until pooled as "normal" or "neutropenic" abscess fluid and used as growth medium in time-kill trials with antibiotics as described below. All animal experiments were approved by the University of Missouri-Kansas City animal care and use committee and were conducted in accordance with guidelines by the Association for Assessment and Accreditation of Laboratory Animal Care.

\subsection{Time Kill of $S$. aureus in Infection Exudates and Broth}

Three growth media were used: sterile-filtered neutronpenic abscess fluid, sterile-filtered normal abscess fluid or MHB. The MIC and MBC of the Staphylococcus aureus strain were determined by the method of Taylor, et al. [11] Stationary phase Staphylococcus aureus $1 \times$ $10^{7} / \mathrm{mL}$ and $100 \mu \mathrm{L}$ containing the minimal bactericidal concentration $(\mathrm{MBC})$ of cephalexin or cefotaxime (or no-antibiotic saline control) were added, and the tubes were incubated at $37^{\circ} \mathrm{C}$. Aliquots were removed for dilution plating on blood agar at $0,3,6$ and $24 \mathrm{hr}$. S. aureus colonies growing on the plates were counted following $24 \mathrm{hr}$ incubation at $37^{\circ} \mathrm{C}$ and bacterial kill at each time point was determined. Each drug time kill was repeated at least 3 times.

\subsection{In Vivo Experiments, Abscess Model}

15 SD male rats, $450+$ gm weight, were implanted intraabdominally with perforated tissue cages as described above. Twenty-four hours following infection, animals were administered cefotaxime $(n=8)$ or cephalexin $(n=$ 7) at $300 \mathrm{mg} / \mathrm{kg} / \mathrm{day}$ subcutaneously. Doses were given $1 / 3$ in a.m. and $2 / 3$ p.m. based on the experimentally determined MIC and MBC of the drugs for the infective isolate of $S$. aureus and historic beta-lactam peak and trough data in rats. Abscesses were sampled on days 1, 3, 5 , and 7 after infection immediately before the a.m. antibiotic dose. Abscess fluid was sonicated at 90 watts for 15 seconds which liberated phagocytized $S$. aureus, dilution plated and bacterial colonies were counted as described above. Aliquots of abscess fluid were individually filtered and frozen for antibiotic assay.

\subsection{Bioassay of Abscess Fluid for Antibiotics}

Bioassay procedures followed published methods [12]. Briefly, duplicate $20 \mu \mathrm{L}$ samples of each abscess fluid, which had been filtered at $0.2 \mu \mathrm{M}$ before storage at $-70^{\circ} \mathrm{C}$, were evaluated for zones of inhibition on Bacillus subtilis pour plates after $24 \mathrm{hr}$ growth. Antibiotic levels were determined by comparing abscess fluid inhibition zones to regression curves of inhibition zones prepared with drug standards run concurrently. 


\subsection{Neutrophil Effect on $S$. aureus PBP 2}

The six lanes on Western blot of $S$. aureus PBPs represent duplicate pairs of $S$. aureus cell walls from organisms exposed or not to neutrophils. S. aureus cell wall protein was isolated from one culture of $S$. aureus, divided then incubated or not with neutrophils. The PBPs were tagged with unlabeled drug $(10 \times$ MIC, cefazolin in this case), followed by electrophoretic separation and detection of the PBP-bound drugs with an anti-beta lactam antibody, appropriate secondary and Western blot development.

\subsection{Statistical Analysis}

Data were expressed as mean $\pm \mathrm{SD}$ and analyses were performed using Statistica (Statsoft, Tulsa OK, USA). Comparisons of two groups utilized Student's t-test. For statistical analysis in the in vivo experiment, end of treatment results were compared to before treatment data. Multiple comparisons used analysis of variance (ANOVA) and the Tukey post-test. Data with a $p$ value of 0.05 or less were considered significantly different, all $\mathrm{p}$ values were two-tailed.

\section{Results}

\subsection{In Vitro and Ex Vivo Experiments}

We sought to determine if the presence of PMNs, which diminishes beta-lactam PBP-2 binding affinity in $S$. aureus, adversely effect the activity of cefotaxime, which binds to PBP-2, compared to cephalexin, which binds to more to PBP 1 and 3 and only minimally to PBP-2. Bacterial killing by cefotaxime and cephalexin were compared in time-kill studies utilizing Clinical and Laboratory Standards Institute culture in the sterile-filtered neutropenic and non-neutropenic abscess fluids. The MIC and $\mathrm{MBC}$ respectively for cefotaxime was 0.5 and 1.0 $\mu \mathrm{g} / \mathrm{ml}$, and for cephalexin was 1.0 and $2.0 \mu \mathrm{g} / \mathrm{ml}$. The concentration of antimicrobial used in the time-kill studies was $1.0 \mu \mathrm{g} / \mathrm{ml}$ cefotaxime and $2.0 \mu \mathrm{g} / \mathrm{ml}$ cephalexin. The $\log _{10} S$. aureus counts following growth in the two media, with or without cefotaxime or cephalexin are shown at 3, 6, and $24 \mathrm{hr}$ in Figures 1 and 2. Cefotaxime, but not cephalexin, was more active in the neutropenic abscess fluid than in the non-neutropenic abscess fluid. Time kill experiments were also performed in MHB. Both cefotaxime and cephalexin killed S. aureus in MHB significantly better than no antibiotic $(p=0.001$, ANOVA and post-test, data not shown), and cefotaxime was more effective than cephalexin when measured after 24-hours incubation (Figures 1, 2). Figure 3 is a Western blot demonstrating the effect of neutrophil incubation on $S$. aureus PBPs.

PBP 2 is virtually eliminated in all neutrophil-incubated

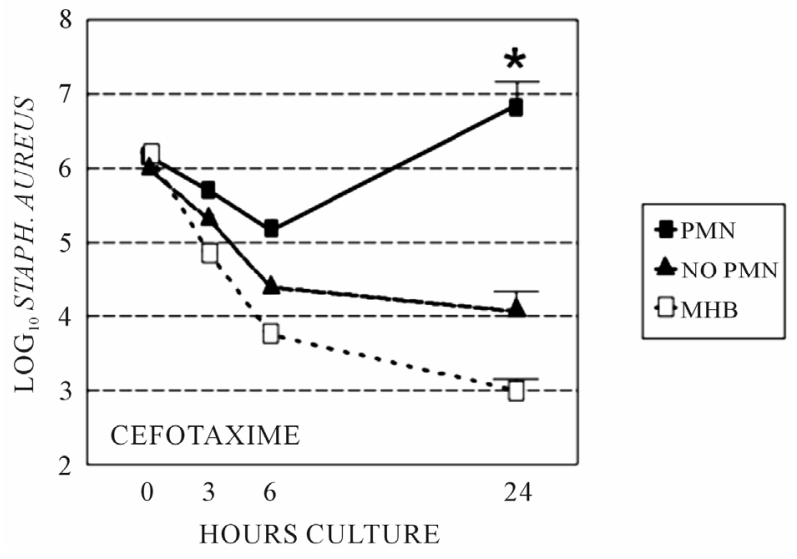

Figure 1. Time kill assays: Stationary phase $S$. aureus kill- ing by cefotaxime, grown in sterile filtered neutropenic ("no PMN") or normal ("PMN") abscess fluids. Killing by cefotaxime in Mueller Hinton broth (MHB) is shown for comparison. *At $24 \mathrm{~h}$, cefotaxime showed greater killing in neutropenic abscess fluid compared to normal abscess fluid, $\mathbf{p}<0.05$.

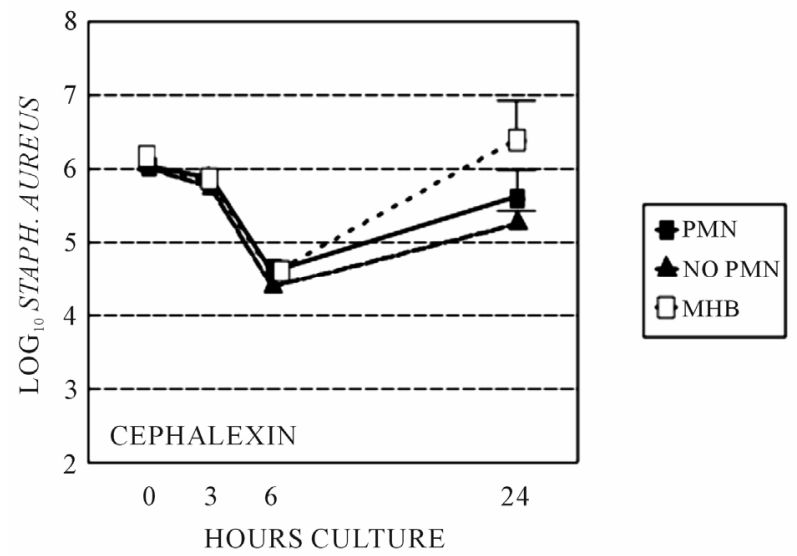

Figure 2. Time kill assays: Stationary phase $S$. aureus killing by cephalexin grown in sterile filtered neutropenic ("no PMN") or normal ("PMN") abscess fluids. Killing by cephalexin in MHB is also shown for comparison. Killing is not significantly different between growth media.

S. aureus cell walls. This occurs regardless of the drug used to label the $S$. aureus preparation.

\subsection{In Vivo Experiments}

To confirm the diminished activity of the PBP-2 binding drug cefotaxime compared to the non-PBP-2 binding drug cephalexin in the presence of PMN containing abscess fluid we studied each antimicrobial agent in a tissue-cage $S$. aureus abscess model, shown in Figure 4.

Over the 7 day treatment time, cephalexin at $300 \mathrm{mg} /$ $\mathrm{kg} / \mathrm{d}$ in divided doses exhibited a $2.1 \pm 1.7 \log _{10}$ drop in bacterial $\mathrm{cfu} / \mathrm{ml}$ from day $0,(\mathrm{p}=0.029$, Student's t-test). Treatment with cefotaxime for 7 days exhibited only a $0.81 \pm 2.5 \log _{10}$ drop in bacterial cfu/ml from day $0(\mathrm{p}=$ 


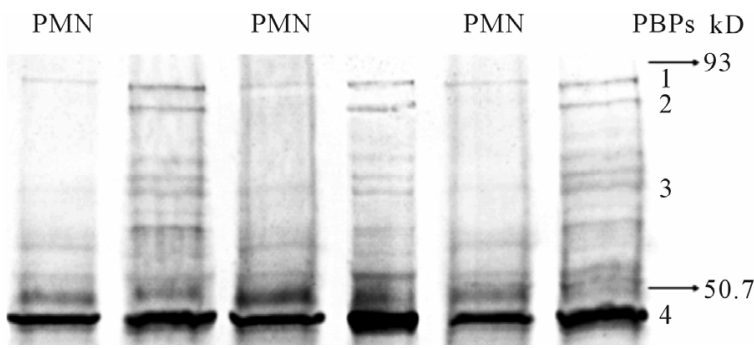

Figure 3. Western blot of the 4 main cell wall penicillin binding proteins (PBPs) of $S$. aureus, incubated or not incubated with neutrophils (PMN). The six lanes on Western blot of $S$. aureus PBPs represent duplicate pairs of $S$. aureus cell walls from organisms exposed or not to PMN. S. aureus cell wall protein was isolated from one culture of $S$. aureus, divided then incubated or not with neutrophils. The PBPs were incubated with unlabeled drug $(10 \times$ MIC), followed by electrophoretic separation and detection of the PBP-bound drugs with an anti-beta lactam antibody, appropriate seconddary and Western blot development. PBP 2 is virtually eliminated in all neutrophil-incubated $S$. aureus cell walls, regardless of the drug used to label the $S$. aureus preparation.

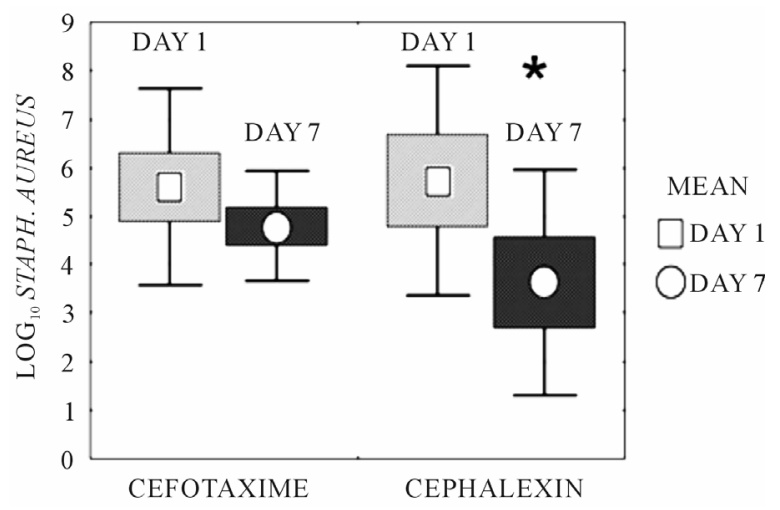

Figure 4. S. aureus concentration in abscesses following in vivo treatment with cefotaxime or cephalexin for seven days. "Days" are the number of days of systemic antimicrobial administration received by the animal from which abscess fluid was harvested. Bacterial counts in the abscess fluid were determined by dilution plating. Boxes represent \pm standard error. Bars represent \pm standard deviation. Mean is identified. Cefotaxime exhibited a $0.81 \pm 2.5 \log _{10}$ drop in bacterial $\mathrm{cfu} / \mathrm{ml}$ over days 0 through $7(p=\mathrm{NS})$. * Cephalexin exhibited a $2.1 \pm 1.7 \log _{10}$ drop in bacterial cfu/ml over days 0 to $7,(p=0.029$, Student's t-test $)$.

NS), confirming the ex vivo experiments. Trough abscess fluid cefotaxime concentration, measured 15 hours after the afternoon dose at day 7 was $1.56 \pm 0.99 \mu \mathrm{g} / \mathrm{ml}$. Trough abscess fluid cephalexin concentration, measured 15 hours after the afternoon dose at day 7 was $1.45 \pm 1.1 \mu \mathrm{g} / \mathrm{ml}$. Both values exceeded the MIC of the test organism.

\section{Discussion}

The novelty of these data is through demonstration by several methods, in vivo, ex vivo and by a new detection process in vitro, that the activity of beta-lactams is diminished by PMNs, and that the loss of activity of beta-lactams in an abscess milieu correlates with the PMN induced alteration of PBP 2. The neutrophil, although vital for the initial control of pyogenic infections, may provide a sanctuary by which $S$. aureus survive [5]. The PMN may then induce substantial changes in the in gene regulation in $S$. aureus. Genes encoding proteins that moderate oxidative stress, virulence, and those involved in metabolism and capsule synthesis are generally upregulated, but the effects vary and some PBP genes are down-regulated after exposure for 30 minutes, and upregulated after exposure for 180 minutes [6]. In our previous experiments utilizing biotinylated ampicillin, betalactam binding to PBP 2 but not PBP 3 was significantly diminished after PMN exposure [4]. Utilizing a differing methodology by labeling with an anti-beta lactam monoclonal antibody [13], the reduction of beta-lactam binding to PBP-2 after PMN exposure was again demonstrated, and shown in Figure 4.

We sought to determine if cefotaxime, a third generation cephalosporin that primarily binds to PBP 1 and 2, has diminished activity in an abscess environment compared to the first generation cephalosporin cephalexin, which primarily binds to PBP 1 and 3, as would be predicted if PMN exposure alters the synthesis of PBP 2 or alters the ability of beta-lactams to bind to the PBP 2 target. Others have noted that strains of $S$. aureus, such as CDC-6, which has an altered PBP-2, is more resistant to cefotaxime as compared to antimicrobials that preferentially bind PBP-3 [14]. Similarly SC 12,700 is an organism with an altered PBP-3 and is more resistant to cephalexin, but is susceptible to cefotaxime [9]. In timekill curves against stationary phase $S$. aureus, cefotaxime was highly active when tested in MHB, and was more active than cephalexin when measured at $24 \mathrm{~h}$. In abscess fluid that was obtained from neutropenic animals, cefotaxime remained highly active in killing stationary phase S. aureus as compared to cephalexin. However in abscess fluid obtained from non-neutropenic animals, cefotaxime had diminished activity in killing $S$. aureus, while cephalexin maintained activity. In the non-neutropenic abscess animal model, cephalexin was more active than cefotaxime after seven days of treatment.

In summary, cephalexin was more active against $S$. aureus exposed to PMNs or PMN products, while cefotaxime was more active against $S$. aureus in the absence of exposure to PMNs. A plausible explanation for these findings is that beta-lactam binding to PBP 2, which is an important target for the activity of cefotaxime but not cephalexin, is altered secondary to PMN exposure, which 
results in loss of activity of cefotaxime but not cephalexin. The present data suggest that the effect observed in vivo was secondary to the neutrophil.

Previous studies have suggested that the stress of increasing concentrations of antimicrobial may produce selection of altered PBPs with resultant loss of antim- icrobial activity [7]. We and others [15] have found that PMN exposure also effects beta-lactam binding to PBPs. The in vivo significance of the altered beta-lactam binding to PBP has not been previously demonstrated. PMN induced alteration of antimicrobial binding proteins with resultant diminished antimicrobial activity defines a novel mechanism of in vivo antimicrobial resistance. Antimicrobial resistance to beta-lactams in stationary phase Streptococcus pyogenes has also been postulated to be secondary to altered PBP production [16]. The mechanisms by which bacterial persist in abscesses despite the presence of antimicrobials at concentrations above the MIC likely remain multifactorial, however included in the potential mechanisms could be alteration of targets of antibacterials by the PMN.

\section{Acknowledgements}

We gratefully acknowledge the contributions of Zarqa Imdad M.D., lab assistants Shena Latcham and Tahira Zufer, and medical students Christine Van Dillen and Nisha Mangalat.

\section{REFERENCES}

[1] D. M. Bamberger. "Outcome of Medical Treatment of Bacterial Abscesses without Therapeutic Drainage: Review of Cases Reported in the Literature," Clinical Infectious Disease, Vol. 23, No. 3, 1996, pp. 592-603. doi:10.1093/clind/23.1.592

[2] D. M. Bamberger, B. L. Herndon, M. Dew, R. P. Chern, H. Mitchell, L. E. Summers, R. F. Marcus, S. C. Kim and P. R. Suvarna, "Efficacies of Ofloxacin, Rifampin, and Clindamycin in Treatment of $S$. aureus Abdominal Abscesses and Correlation with Results of an in Vitro Assay of Intracellular Bacterial Killing," Antimicrobial Agents \& Chemotherapy, Vol. 41, No. 5, 1997, pp. 1178-1181.

[3] D. M. Bamberger and B. L. Herndon, "Bactericidal Capacity of Neutrophils in Rabbits with Experimental Acute and Chronic Abscesses," Journal of Infectious Disease, Vol. 162, No. 1, 1990, pp. 186-192. doi:10.1093/infdis/162.1.186

[4] D. M. Bamberger, B. L. Herndon, J. Fitch, A. Florkowski and V. Parkhurst, "The Effect of Neutrophils on Cefazolin Activity and Penicillin Binding Proteins in Staphylococcus aureus Abscesses," Antimicrobial Agents \& Chemotherapy, Vol. 46, No. 9, 2002, pp. 2878-2884. doi:10.1128/AAC.46.9.2878-2884.2002

[5] H. D. Gresham, J. H. Lowrance, T. E. Caver, B. S. Wilson, A. L. Cheung and F. P. Lindberg, "Survival of Staphylococcus aureus inside Neutrophils Contributes to Infec- tion," Journal of Immunology, Vol. 164, No. 7, 2000, pp. 3713-3722.

[6] J. M. Voyich, K. R. Braughton, D. E. Studevant, A. R. Whiney, B. Said-Salim, S. F. Porcella, et al., "Insights into Mechanisms Used by Staphylococcus aureus to Avoid Destruction by Human Neutrophils," Journal of Immunology, Vol. 175, No. 6, 2005, pp. 3907-3919.

[7] S. Turk, O. Verlaine, T. Gerards, M. Zivec, J. Humljan, I. Sosic, A. Amoroso, A. Zervosen, A. Luxen, B. Joris and S. Gobec, "New Noncovalent Inhibitors of Penicilin-Binding Proteins from Penicillin-Resistant Bacteria," PLOS ONE, Vol. 6, No. 5, 2011, Article ID: e19418. doi:10.1371/journal.pone.0019418

[8] S. Bobba and W. G. Gutheil, "Multivariate Geometrical Analysis of Ctralytic Residues in the Penicillin-Binding Proteins," The International Journal of Biochemistry \& Cell Biology, Vol. 43, No. 10, 2011, pp. 1490-1499. doi:10.1016/j.biocel.2011.06.012

[9] N. H. Georgopapadakou, S. A. Smith, and D. P. Bonner, "Penicillin-Binding Proteins in a Staphylococcus aureus Strain Resistant to Specific $\beta$-Lactam Antibiotics," Antimicrobial Agents \& Chemotherapy, Vol. 22, No. 1, 1982, pp. 172-175.

[10] E. Tonin and A. Tomasz. "Beta-Lactam-Specific Resistant Mutants of Staphylococcus aureus," Antimicrobial Agents \& Chemotherapy, Vol. 30, No. 4, 1986, pp. 577-583.

[11] P. C. Taylor, F. D. Schoenknecht, J. C. Sherris and E. C. Linner, "Determination of Minimum Bactericidal Concentrations of Oxacillin for Staphylococcus aureus: Influence and Significance of Technical Factors," Antimicrobial Agents \& Chemotherapy, Vol. 23, No. 1, 1983, pp. 142-150.

[12] National Committee for Clinical Laboratory Standards, "Performance Standards for Antimicrobial Disk Susceptibility Tests: Approved Standard-11th Edition," Vol. 32, No. 1, Clinical and Laboratory Standards Institute, Wayne, USA, 2012.

[13] A. Koomer, T. Quinn, D. Bamberger and B. L. Herndon, "Neutrophil-Antimicrobial Interaction in the Established Infection: Effect on Staphylococcus aureus," Journal of Infection, Vol. 52, No. 5, 2006, pp. 288-320. doi:10.1016/j.jinf.2005.07.017

[14] A. Tomasz, H. B. Drugeon, H. M. de Lencastre, D. Jabes, L. McDougall and J. Bille, "New Mechanism for Methicillin Resistance in Staphylococcus aureus: Clinical Isolates That Lack the PBP 2a Gene and Contain Normal Penicillin-Binding Proteins with Modified PenicillinBinding Capacity," Antimicrobial Agents \& Chemotherapy, Vol. 33, No. 11, 1989, pp. 1869-1874.

[15] R. M. Rakita, B. R. Michel, and H. Rosen, "Inactivation of Escherichia Coli Penicillin-Binding Proteins by Human Neutrophils," Infection and Immunity, Vol. 62, No. 1, 1994, pp. 162-165.

[16] D. L. Stevens, S. Yan and A. E. Bryant, "PenicillinBinding Protein Expression at Different Growth Stages Determines Penicillin Efficacy in Vitro and in Vivo: An Explanation for the Inoculum Effect," Journal of Infectious Diseases, Vol. 167, No. 6, 1993, pp. 1401-1405. doi:10.1093/infdis/167.6.1401 